\title{
Epitaxial oxide thin films by pulsed laser deposition: Retrospect and prospect
}

\author{
M S HEGDE \\ Solid State and Structural Chemistry Unit, Indian Institute of Science, \\ Bangalore 560012, India \\ e-mail: mshegde@sscu.iisc.ernet.in
}

\begin{abstract}
Pulsed laser deposition (PLD) is a unique method to obtain epitaxial multi-component oxide films. Highly stoichiometric, nearly single crystal-like materials in the form of films can be made by PLD. Oxides which are synthesized at high oxygen pressure can be made into films at low oxygen partial pressure. Epitaxial thin films of high $T_{c}$ cuprates, metallic, ferroelectric, ferromagnetic, dielectric oxides, superconductor-metal-superconductor Josephson junctions and oxide superlattices have been made by PLD. In this article, an overview of preparation, characterization and properties of epitaxial oxide films and their applications are presented. Future prospects of the method for fabricating epitaxial films of transition metal nitrides, chalcogenides, carbides and borides are discussed.
\end{abstract}

Keywords. Pulsed laser deposition; epitaxial films; superconducting oxide films; metallic oxides; ferromagnetic oxide films; superlattices.

\section{Introduction}

Among the large number of processes to fabricate thin films of materials, pulsed laser deposition (PLD) has emerged as a unique method to obtain epitaxial and nearly single crystal like thin films of multi-component oxides. Epitaxial thin films of superconducting high $T_{c}$ cuprates, metallic, ferroelectric, ferromagnetic, dielectric oxides and their multilayers have been made by this method ${ }^{1}$. Conceptually and operationally, PLD is a simple technique. A laser beam enters a vacuum chamber through a window and impinges on the target material to be deposited. The 20- to 30nanosecond wide laser pulse is focused to an energy density of 1 to $5 \mathrm{~J} / \mathrm{cm}^{2}$ to vaporize a few hundred angstroms of surface material. The vapour contains neutral atoms, positive and negative ions, electrons, molecules and molecular ions, free radicals of the target material in their ground and excited states. These particles emit radiation. These particles acquire kinetic energy of 1 to $5 \mathrm{eV}$ and move in the direction perpendicular to the target. The sum total of all the particles corresponds to the chemical composition of the target. They deposit on a substrate generally heated to a temperature to grow crystalline film.

Laser beam-solid interaction is a complex phenomenon. Energy of the laser-photon beam is absorbed by the solid surface leading to electronic excitation. Since thermal conductivity of the target solid is far too low to dissipate energy in the short period of the laser pulse, photon energy is converted into thermal and chemical energy. Due to this, temperature of the order of $3500{ }^{\circ} \mathrm{C}$ is reached in a small volume of the target 
leading to instantaneous evaporation, ionization and decomposition of the material. The moving front of such a collection of particles constituting a plasma is called a plume. These are collected on the substrate to form a film.

Although the laser evaporation method was used to make films even in the $1960 \mathrm{~s}$,

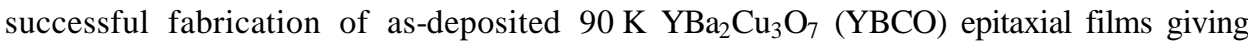
$10^{6} \mathrm{~A} / \mathrm{cm}^{2}$ critical current during late 1987 and early 1988 lead to rapid development of this method ${ }^{2,3}$. Since then, PLD has been extensively used to fabricate epitaxial films of superconducting, metallic, ferroelectric, ferromagnetic oxides and their multilayers. We have set up a PLD facility and during the last 12 years, epitaxial films of a variety of materials have been made here. In this article, we present an overview of preparation and properties of epitaxial thin films and multilayers by PLD and discuss future prospect of this method.

\section{Growth of epitaxial films}

Generally, $\mathrm{KrF}(248 \mathrm{~nm})$ and $\mathrm{XeCl}(308 \mathrm{~nm}) \mathrm{UV}$ excimer lasers are used in PLD. Schematic diagram of a PLD system is shown in figure 1 . The system consists of a vacuum chamber which is evacuated by a diffusion pump to $10^{-6}$ Torr. The laser beam incident at an angle of $45^{\circ}$ to the target surface is focused on to the rotating target, which is an $18 \mathrm{~mm}$ diameter pellet of the material whose film is to be deposited. Laser beams of energy 300 to $400 \mathrm{~mJ}$ are focused to a size such that energy density is maintained between 1 to $5 \mathrm{~J} / \mathrm{cm}^{2}$. The target holder is designed to have three targets so that multilayer film can be grown in situ. The substrate on which the film is to be grown is placed opposite to the target at a distance of 3.5 to $4 \mathrm{~cm}$. Substrate can be heated to $800^{\circ} \mathrm{C}$. Gas inlet facility is provided so that films can be grown in oxygen or any other gaseous environment. Generally, 300 to 400 mTorr gas pressure is employed during the growth of the film. For growing films other than oxides, base vacuum needed is $10^{-9}$ Torr.

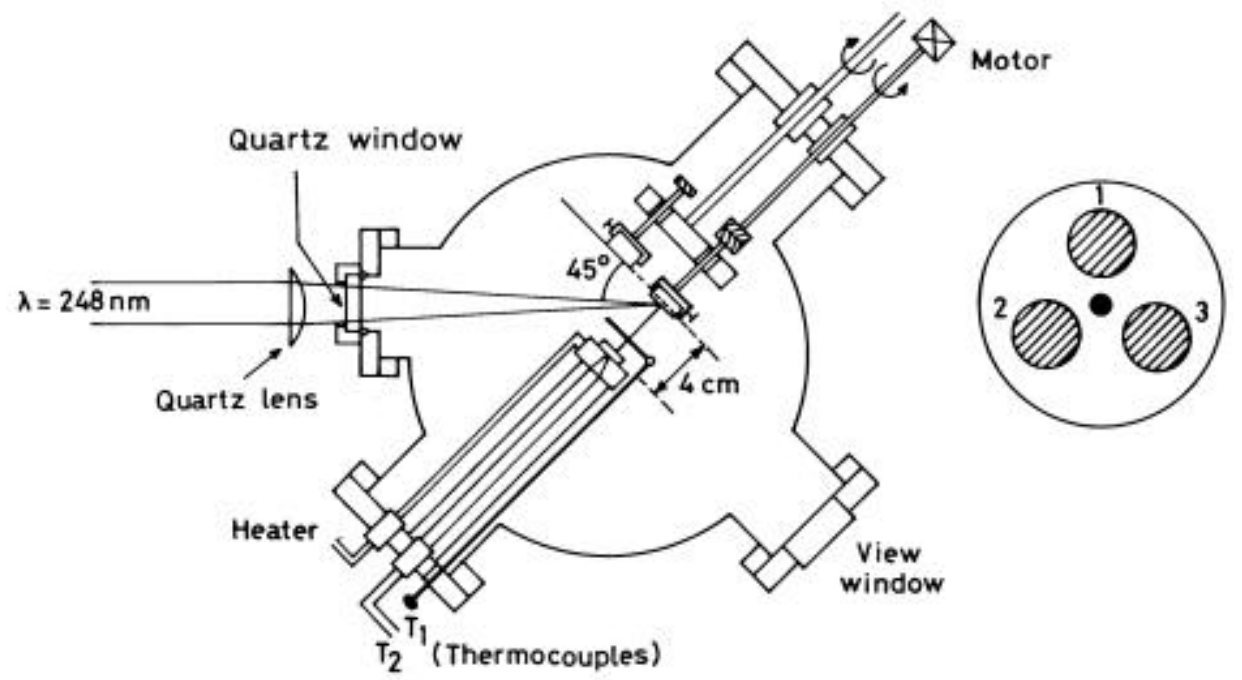

Figure 1. Schematic diagram of a pulsed laser deposition (PLD) system. Positions of three targets in the holder are also shown. 


\subsection{Substrates}

Most of the materials so far grown into films by PLD are perovskite-related oxides. Therefore to grow these oxide films, lattice-matched single crystal substrates are necessary. Commonly used substrates are $\mathrm{SrTiO}_{3}, \mathrm{LaAlO}_{3}, \mathrm{MgO}, \mathrm{ZrO}_{2}$ and sapphire which can be cut in [100], [110] or [111] direction. As thermal expansion coefficients of oxides are close to those of the substrates, oxide films on these substrates do not crack. Since Si is a covalent material, the probability of ions sticking on it is low. There is no ionic bonding between $\mathrm{Si}$ and the oxides. Also there is large thermal mismatch and therefore growing epitaxial films on single crystal silicon substrate has not been successful. However, $\mathrm{BaTiO}_{3}$ grown on $\mathrm{MgAl}_{2} \mathrm{O}_{4}$ on $\mathrm{Si}(100)$ provides thermal expansion graded substrate for growing perovskite-related oxide films including $\mathrm{YBa}_{2} \mathrm{Cu}_{3} \mathrm{O}_{7}(\mathrm{YBCO})$.

\subsection{Deposition parameters}

Target-substrate distance, partial pressure of oxygen, substrate temperature, laser pulse energy density and pulse repetition rate are the main five parameters that need to be optimized to get the desired films.

It is important to recognize that highly stoichiometric, nearly single crystal like epitaxial film is aimed for in the PLD method. Streams of ions, or neutral atoms arrive at the surface of the substrate. The substrate surface consisting of positive and negative ions attracts ions of opposite charge thus initiating the layer growth. Layer by layer growth of crystal starts with the mobility of ions on the surface aided by their own kinetic energy and the substrate temperature. The process can be compared with the growth of ionic crystals from its solution on a seed crystal. Preferred growth rate is of the order of $1 \AA$ per pulse.

\section{Characterization of films}

X-ray diffraction (XRD) remains the main characterization tool for epitaxial films. Normal $\boldsymbol{\theta}-2 \boldsymbol{\theta}$ scan gives the out-of-plane (generally $c$ ) parameter of the film. For example, for YBCO films, (001) lines provide the $c$ parameter. For perovskite-related oxides, therefore, only one parameter is obtained. This is depicted in figure 2 where (001) lines of $\mathrm{YBa}_{2} \mathrm{Cu}_{3} \mathrm{O}_{7}$ (YBCO) grown in situ on $\mathrm{LaNiO}_{3}$ (LNO) over $\mathrm{SrTiO}_{3}(100)$ substrate. Only the $(001)$ lines of YBCO, LNO and those from the substrate are seen. The other two parameters $a$ and $b$ need to be obtained from grazing incidence (inplane) diffraction studies. Lattice parameters from in-plane diffraction study are rare in the literature. Lattice parameters of the film agreeing well with their bulk solid confirm the phase formation in the film.

Sitting on a single $(001)$ line and a $\Omega$ scan will provide information on the quality of the crystal. Such a scan is called rocking curve. Higher widths of the diffraction line in the $\Omega$ scan indicate poor crystallinity.

Epitaxial growth needs to be confirmed from 0 to $360^{\circ} \Phi$ scans. For example, epitaxial growth of YBCO film on $\mathrm{LaNiO}_{3}$ film which is grown on $\mathrm{LaAlO}_{3}(100)$ is demonstrated in figure 3. Here, growth of $\mathrm{YBCO}$ and $\mathrm{LaNiO}_{3}$ in $(001)$ direction is first seen from the normal $\theta-2 \theta$ scans and $\Phi$ scans are recorded for a diffraction line of $\mathrm{LaAlO}_{3}, \mathrm{LaNiO}_{3}$ and $\mathrm{YBCO}$ individually in a single setting of the diffractometer. 


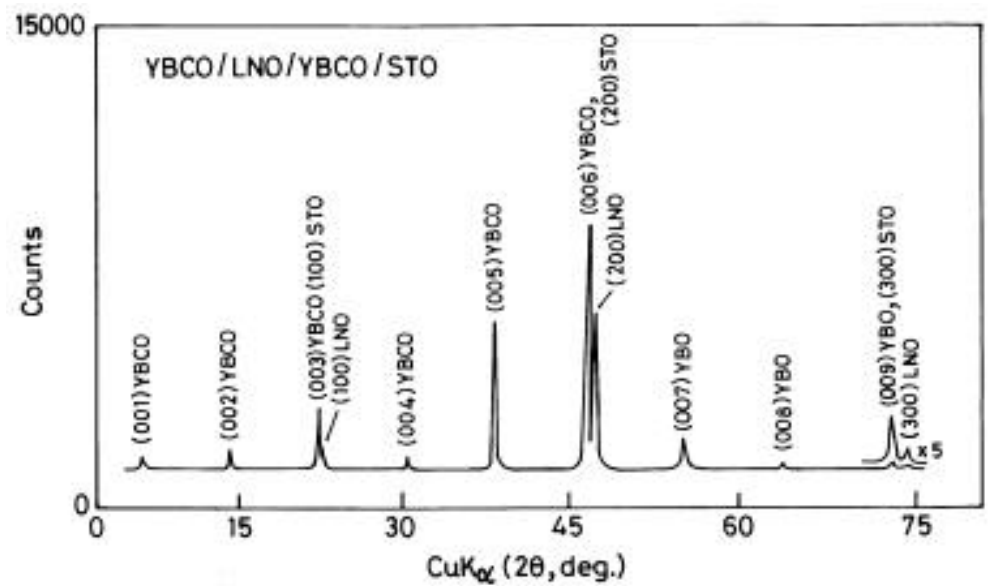

Figure 2. $\theta-2 \theta \mathrm{XRD}$ pattern of $\mathrm{YBCO} / \mathrm{LNO} / \mathrm{YBCO}$ heterostructure on $\mathrm{LAO}$ (1 000$)$ substrate.

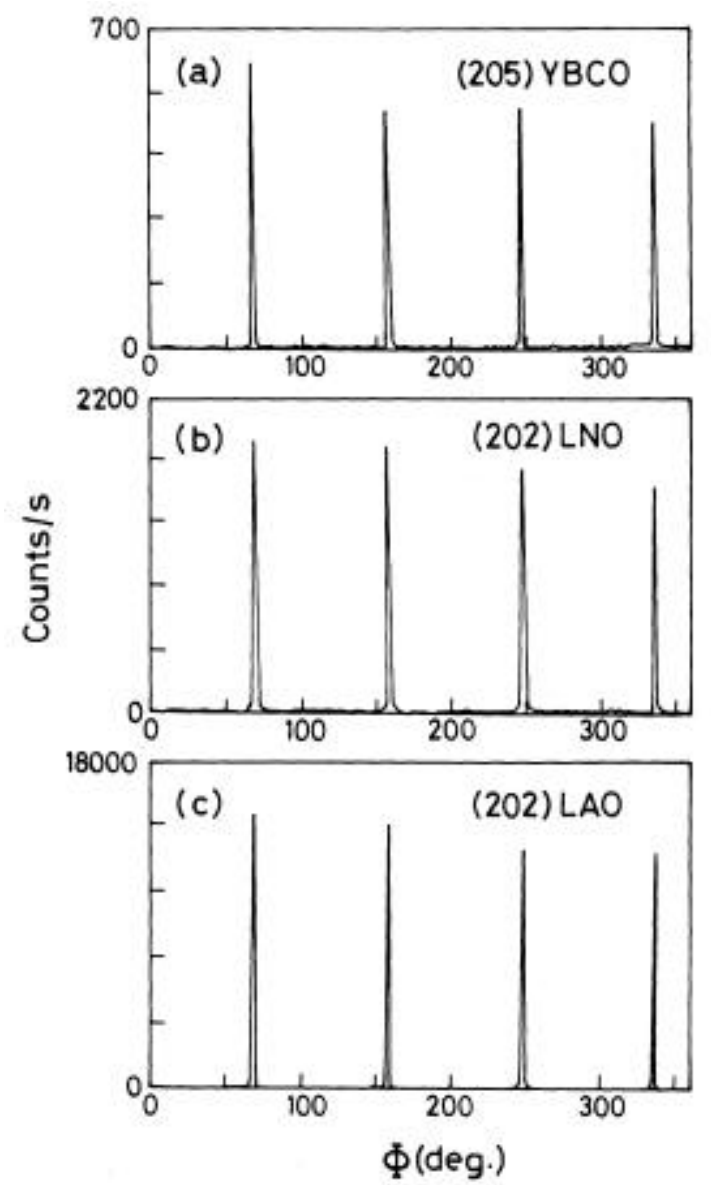

Figure 3. In-plane X-ray diffraction $\Phi$-scan of (a) YBCO (20 5), (b) LNO (202), and (c) LAO (202) peaks of YBCO/LNO/LAO thin films. 
Coincidence of the $\Phi$ angle of the substrate with the film confirms the epitaxial relation. Four lines separated by $90^{\circ}$ in the $\Phi$ scan at the same $\Phi$ angle confirm the growth of LNO and 123 in $C_{4}$ symmetry ${ }^{4}$. Appearance of peaks at $45^{\circ}$ separation would show $45^{\circ}$ rotation of the material in the $a-b$ plane. Generally, a rotation of $45^{\circ}$ with respect to basal plane is observed for perovskite films on cubic $\mathrm{MgO}$ and $\mathrm{ZrO}_{2}$ substrates.

XRD is a basic method to characterize artificially made layered thin film structures. In the $\boldsymbol{\theta}-2 \boldsymbol{\theta}$ scan, satellite peaks observed on either side of the main Bragg reflections (growth direction) confirm the layered nature of the film. The modulation wavelength (sum of the consecutive layer thickness) $D$ can be calculated from the satellite positions using the equation, $D=\lambda 2\left[1 /\left(\sin \theta_{1}-\sin \theta_{2}\right)\right]$, where $\lambda$ is the $X$-ray wavelength and $\theta_{1}$ and $\boldsymbol{\theta}_{2}$ are Bragg angles for the consecutive diffraction maxima ${ }^{5}$.

Cross-section transmission electron microscopy of the films would give structure as well as ordering at the interface. This is a specialized technique for structural elucidation of epitaxial films.

Other characterization tools are resistivity, magnetoresistance and magentic properties. The electronic structure of the film is studied by X-ray photoelectron spectroscopy (XPS). Surface smoothness and morphology can be investigated by scanning electron microscopy (SEM), atomic force microscopy (AFM), scanning tunneling microscopy (STM). Structure-properties of epitaxial film in comparison with the polycrystalline and single crystal material constitute complete study of the film.

\section{Epitaxial films of high $T_{c}$ cuprates}

Supercoductivity was discovered in $\mathrm{La}_{1-x} \mathrm{Ba}(\mathrm{Sr})_{x} \mathrm{CuO}_{4}$ systems in 1986 and subsequent observation of superconductivity at $90 \mathrm{~K}$ in $\mathrm{YBa}_{2} \mathrm{Cu}_{3} \mathrm{O}_{7}$ (YBCO) started a new era of solid state chemistry and physics. With the growth of epitaxial YBCO films on $\mathrm{SrTiO}_{3}$ (100) showing critical current, $J_{c}$, in the order $10^{6} \mathrm{~A} / \mathrm{cm}^{2}$, by Praveen Chaudhari ${ }^{6}$ at IBM, growing epitaxial oxide thin films gained importance. Venky Venkatesan and his group at Bellcore first made YBCO films by PLD $^{2}$. These films were to be annealed in oxygen at $450^{\circ} \mathrm{C}$. By providing required amount of oxygen during the film growth, as deposited YBCO film showed zero resistivity at $90 \mathrm{~K}^{3}$. This was the beginning of the new era of PLD.

Although a series of $\mathrm{Bi}, \mathrm{Tl}$ and $\mathrm{Hg}$ based cuprates are known today ${ }^{7}$, applications have taken off with YBCO. The preparation of YBCO epitaxial thin films on different substrates has been standardized. Superconducting wires of micron width are routinely patterned. For microwave transmission applications, it is not sufficient if $J_{c}$ and $T_{c}$ are high. Surface conductivity of the high $T_{c}$ superconducting materials should also be high without any microwave loss due to the substrate. This is achieved by the addition of $7 \% \mathrm{Ag}$ to the YBCO while making the film ${ }^{8,9}$. For microwave transmission applications, $\mathrm{SrTiO}_{3}$ and $\mathrm{LaAlO}_{3}$ are not suitable substrates due to high loss. YBCO with $7 \% \mathrm{Ag}$ film grown on sapphire gave high performance in terms of low loss and high surface conductivity ${ }^{10}$. $\mathrm{Ag}$ is shown to bring in extra oxygen during the YBCO growth $^{11}$. Silver fills the voids created due to the columnar growth and provides smooth surface. Ag also provides resistance to corrosion and degradation that becomes an important asset for the device applications.

Complete substitution of $\mathrm{Pr}$ for $\mathrm{Y}$ in $\mathrm{YBCO}$ is possible but $\mathrm{PrBa}_{2} \mathrm{Cu}_{3} \mathrm{O}_{7}$ (PBCO) is non-superconducting. However, it is possible to substitute partially and continuous 
decrease of $T_{c}$ is seen with increasing $x$ in $\mathrm{Y}_{1-x} \mathrm{Pr}_{x} \mathrm{Ba}_{2} \mathrm{Cu}_{3} \mathrm{O}_{7}$. A series of Pr-substituted YBCO films have been fabricated by PLD and $T_{c}$ and $J_{c}$ were measured. We have shown that for $x<0.05, J_{c}$ can be higher than YBCO due to flux pinning but for higher $x, J_{c}$ decreases exponantially ${ }^{12}$.

Ever since the discovery of high $T_{c}$ cuprates there have been enormous attempts to use them for different applications. High $T_{c}$ materials win over conventional superconductors on device-operating temperature as they work at liquid nitrogen temperatures. When a superconductor is separated by a small distance (or by an insulating material) supercurrents can tunnel from one side to the other showing nonlinear current-voltage $(I-V)$ characteristics. This kind of structure was first theoretically studied by Josephson and later proved by Rowel and Anderson experimentally in conventional superconductors. When a normal metal is sandwiched between the superconductors it shows a proximity effect and also shows nonlinear $I-V$ characteristics. Characteristics of superconductor-insulator-superconductor (SIS) and superconductor-normal metal-superconductor (SNS) Josephson junctions have been studied by De Gennes and Tinkham. The first such device was fabricated with $\mathrm{YBCO} / \mathrm{PBCO} / \mathrm{YBCO}$ and the Josephson effect was seen ${ }^{13}$. Surprisingly it showed only SNS type behaviour. Even today, SIS-type Josephson junctions have not been made with high $T_{c}$ superconductors. Therefore, studies with metallic oxide as the barrier layer were carried out. $\mathrm{LaNiO}_{3}$ (LNO) is one such metallic oxide that can be used as the metal barrier layer in SNS junctions. Typical $I-V$ characteristics and the oscillation of $I_{c}$ with magnetic field from $\mathrm{YBCO} / \mathrm{LNO} / \mathrm{YBCO}$ trilayer junction fabricated here is shown in figure 4 . Significantly high $I_{c} R_{n}$ product $(1 \mathrm{mV})$ is achieved with LNO as the normal metal barrier layer ${ }^{14}$.

Many groups have fabricated Josephson junctions by using YBCO superconductors and the best performance has been observed at $77 \mathrm{~K}$. Combining two Josephson junctions in a superconducting loop shows current interference analogous to optical counter part of Young's double slit experiment. These structures termed as SQUID (superconducting quantum interference device) are very powerful in determining very small magnetic fields of the order of $10^{-13}$ tesla. This new class of Josephson junctions is attractive for novel phase devices. Using high- $T_{C}$ single crystals, corner junctions and $\pi$ SQUIDs were fabricated between YBCO and conventional superconductors ${ }^{15}$. Josephson junctions formed between a conventional $S$-wave superconductor and the (110) face of a $d_{x-y}^{2}{ }^{2}$-wave high $T_{c}$ cuprate have been proposed for the realization of qbits, the key element for the quantum computation by Ioffe et $^{16}{ }^{16}$.

\section{Metallic oxide films}

While superconducting oxide films are important, for many applications such as normal metal for SNS-type Josephson junction and metallic electrodes for making electrical contacts, growing epitaxial metallic oxide films itself became an important area of research. The idea of making electrical contact by an oxide metal to a superconducting or insulating oxide material is a new concept. Just as metalmetal contact is achieved by the formation of alloy at the interface, metallic oxide-insulating oxide form an ionic bond at the interface. Indeed, Ramesh et al ${ }^{17}$ showed $10^{12}$ times switching in a ferroelectric device when $\mathrm{Bi}_{4} \mathrm{Ti}_{3} \mathrm{O}_{12}$ film is connected with $\mathrm{La}_{0.67} \mathrm{Sr}_{0.33} \mathrm{CoO}_{3}$ metallic oxide electrodes instead of gold which gave only $10^{6}$ times switching. $\mathrm{LaNiO}_{3}, \mathrm{LaCuO}_{3}, \mathrm{SrRuO}_{3}, \mathrm{La}_{0.66} \mathrm{Sr}_{0.33} \mathrm{CoO}_{3}, \mathrm{SrMoO}_{3}, \mathrm{Na}_{x} \mathrm{WO}_{3}$, 

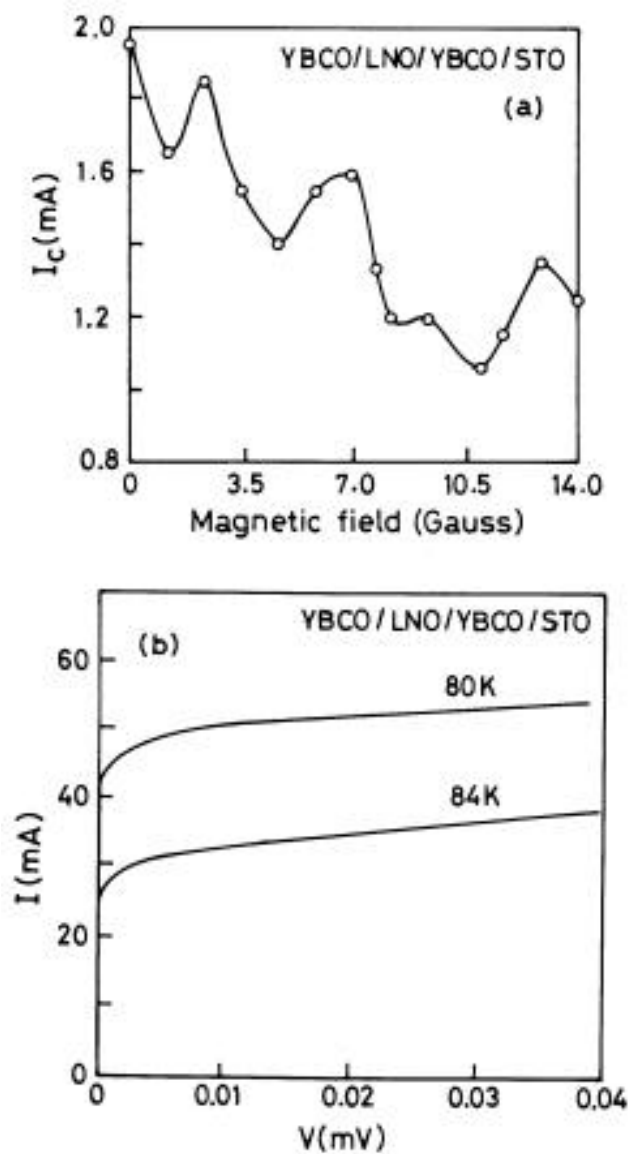

Figure 4. Illustration of DC Josephson effect of $\mathrm{YBCO} / \mathrm{LNO} / \mathrm{YBCO} / \mathrm{STO}$ Josephson junction. (a) Oscillations of critical current in the applied magnetic field can be seen from the plot. (b) $I-V$ characteristics at different temperatures.

$\mathrm{La}_{4} \mathrm{BaCu}_{5} \mathrm{O}_{13}$ are some of the oxides which show metallic behaviour down to measurable temperature say 1 to $2 \mathrm{~K}$. Epitaxial films of $\mathrm{LaNiO}_{3}(\mathrm{LNO})$ grown by PLD have shown high promise for all these applications ${ }^{18-20}$.

$\mathrm{BaPbO}_{3}$ is a metallic oxide having lattice parameter of $4.27 \AA$. Thin films on $\mathrm{LaAlO}_{3}$ show growth along [100] and [1 10] direction as can be seen from figure 5a. Metallic property in resistance $(R)$ vs temperature $(T)$ is shown in figure $5 \mathrm{~b} . \mathrm{BaPbO}_{3}$ can be a good lattice matched metal electrode for ferroelectric PZT and PLZT oxide materials.

$\mathrm{La}_{4} \mathrm{BaCu}_{5} \mathrm{O}_{13}$ (LBCO) was considered a three-dimensional metallic oxide. We have recently shown that this is a rare material which shows metallic behaviour in the $c$ direction and semiconducting behaviour in the $a-b$ plane. This is demonstrated employing LNO as an electrode. LBCO film was grown on LNO and also capped with LNO layer. $R$ vs $T$ plot along $a-b$ plane and $c$ direction were measured and in figure 6 , anisotropic conductivity of this material is presented ${ }^{21}$. 

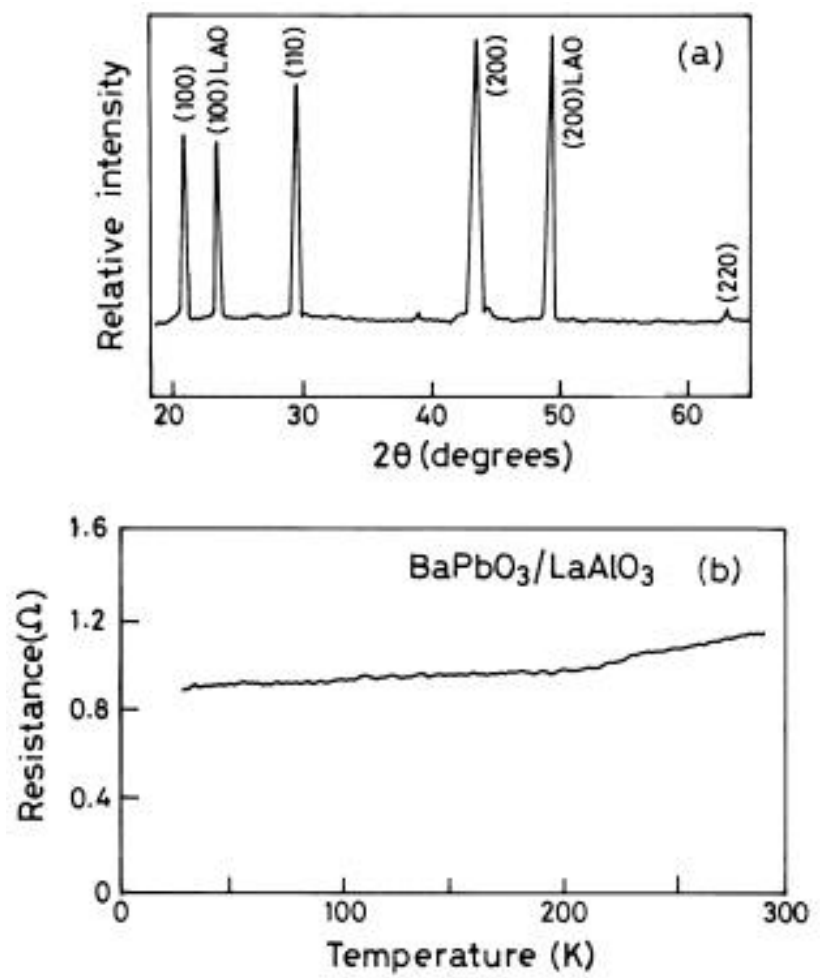

Figure 5. (a) $\theta-2 \theta \mathrm{XRD}$ pattern of $\mathrm{BaPbO}_{3}$ shows $\left(\begin{array}{lll}1 & 0 & 0\end{array}\right)$ and $\left(\begin{array}{lll}1 & 1 & 0\end{array}\right)$ orientations on $\mathrm{LaAlO}_{3}\left(\begin{array}{lll}1 & 0 & 0\end{array}\right)$ substrate. (b) Resistance vs temperature plot of $\mathrm{BaPbO}_{3}$.

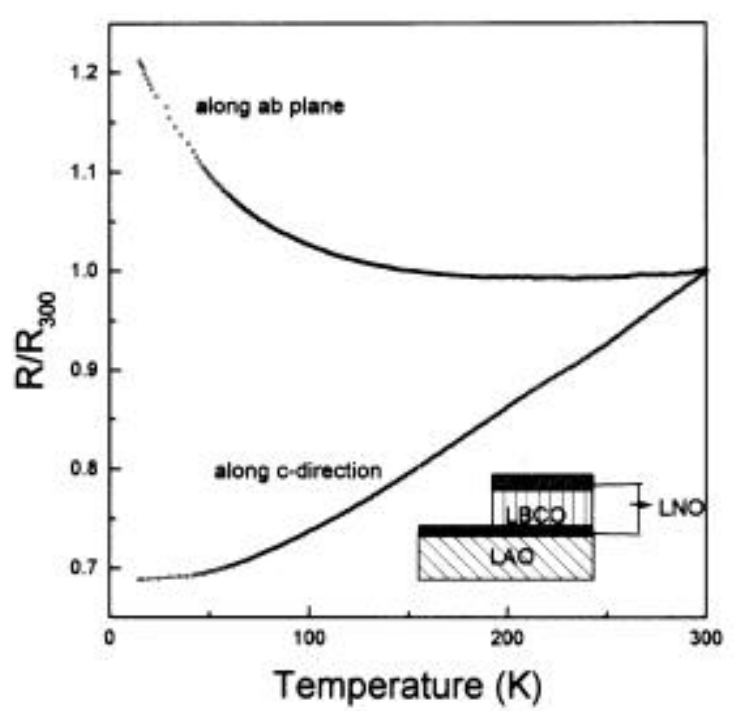

Figure 6. Normalized resistance vs temperature plot of LBCO measured along $a-b$ plane and $c$-directions are shown. Inset shows the schematic representation of the thin film structure employed for the $c$-direction resistance measurement. 
Another aspect of PLD method is the possibility of stabilizing transition metals in higher oxidation states. Stabilization of $\mathrm{Cu}$ in +2 and +3 oxidation states is the main reason for the success of PLD in obtaining as deposited $90 \mathrm{~K} \mathrm{YBCO} \mathrm{films.} \mathrm{PrNiO}_{3}$, $\mathrm{NdNiO}_{3}$ and $\mathrm{SmNiO}_{3}$ solids can be synthesized only at high oxygen pressure. But films of these oxides can be grown at PLD conditions ${ }^{22}$. Further, these films are grown from mixed oxide pellets instead of stoichiometric oxide pellets.

$\mathrm{LaNiO}_{3}$ has been employed as an electrode for metallic contact in ferroelectric switching devices of $\mathrm{Bi}_{2} \mathrm{VO}_{5.5}$ oxide ${ }^{23,24}$ and $\mathrm{Bi}_{4} \mathrm{Ti}_{3} \mathrm{O}_{12}$ oxides. Typical $\mathrm{X}$-ray diffraction patterns and the ferroelectric hysteresis loops are presented in figure 7.

\section{Magnetic oxides films and colossal magnetoresistance}

$\mathrm{LaMnO}_{3}$ is an antiferromagnetic oxide crystallizing in orthorhombic structure. Alkali and lead ion substituted $\mathrm{La}_{1-x} \mathrm{~A}_{x} \mathrm{MnO}_{3}(\mathrm{~A}=\mathrm{Ca}, \mathrm{Sr}, \mathrm{Ba}, \mathrm{Pb})$ showed metallic and ferromagnetic properties. The structure changed from orthorhombic to rhombohedral to cubic symmetry with increase in $x$ up to $0 \cdot 33$. This was known even in the $1950 \mathrm{~s}^{25}$. Epitaxial films of $\mathrm{La}_{0.67} \mathrm{Ba}_{0.33} \mathrm{MnO}_{3}$ (LBMO) were made by PLD in 1993 and

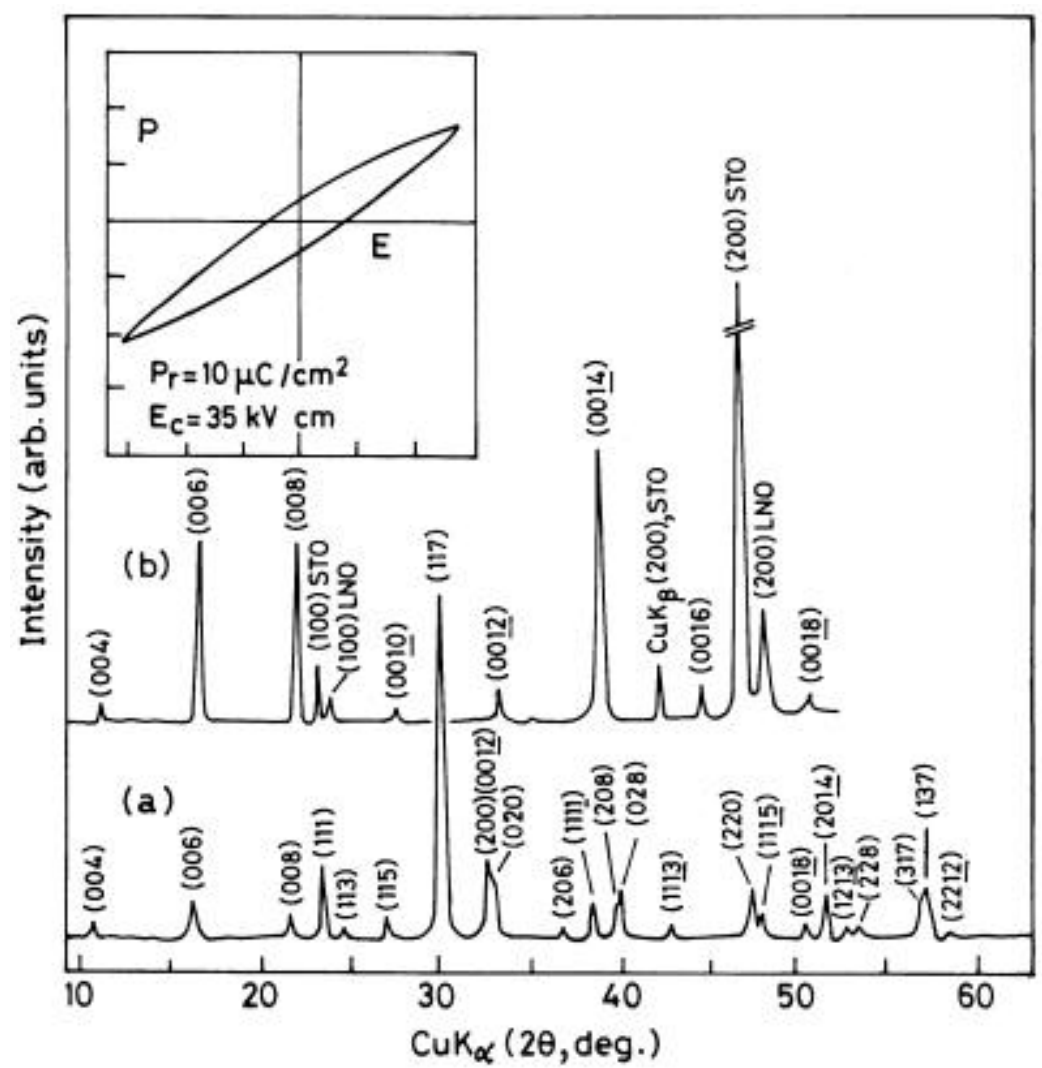

Figure 7. XRD patterns of (a) bulk polycrystalline $\mathrm{Bi}_{4} \mathrm{Ti}_{3} \mathrm{O}_{12}$ and (b) $\mathrm{Bi}_{4} \mathrm{Ti}_{3} \mathrm{O}_{12}$

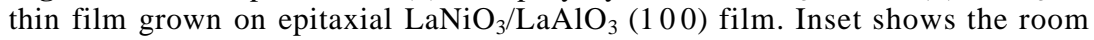
temperature ferroelectric hysteresis loop of $\mathrm{Au} / \mathrm{Bi}_{4} \mathrm{Ti}_{3} \mathrm{O}_{12} / \mathrm{LaNiO}_{3} / \mathrm{LaAlO}_{3}(100)$. 
observation of giant magnetoresistance opened up a new area of research called colossal magnetoresistance $(\mathrm{CMR})^{26} . R_{H}$ and $R_{0}$ are the resistances of the film in presence and absence of external magnetic field respectively which are measured as a function of temperature. The ratio $\left[R_{H}-R_{0}\right] / R_{0} \times 100$ is defined as CMR. Over $60 \%$ CMR was seen in LBMO films at room temperature at a magnetic field of 6 tesla. Observation of such large CMR was considered to have technological applications. Soon, epitaxial films of $\mathrm{La}_{0.67} \mathrm{Ca}_{0.33} \mathrm{MnO}_{3}$ (LCMO) ${ }^{27}, \mathrm{La}_{0.67} \mathrm{Sr}_{0.33} \mathrm{MnO}_{3}$ (LSMO) ${ }^{28}$ films were grown and CMR of these materials are studied.

In our laboratory, we have made epitaxial films of $\mathrm{La}_{1-x} \mathrm{~Pb}_{x} \mathrm{MnO}_{3}, \mathrm{La}_{1-x} \mathrm{~Pb}_{x}$ $\mathrm{Mn}_{1-y} \mathrm{Ti}_{y} \mathrm{O}_{3}, \quad \mathrm{La}_{1-x} \mathrm{MnO}_{3}, \quad \mathrm{La}_{1-x} \mathrm{~Pb}_{x} \mathrm{MnO}_{3-y}$, and studied their structure and $\mathrm{CMR}$ properties ${ }^{29-32} \cdot \mathrm{Cd}^{2+}$ ion can also be substituted for La to a limited extent $(x=0 \cdot 25$ to 0.33 ) and both in the solid state and in thin film form it behaves similar to Ca ionsubstituted $\mathrm{LaMnO}_{3}{ }^{33}$. With $\mathrm{Ti}^{4+}$ ion substitution, ferromagnetic transition temperature $T_{c}$ decreases and increase in CMR at the cost of temperature is observed. Similarly, oxygen-deficient films show an increase in resistivity, decrease in $T_{c}$ and an increase in magnetoresistance.

Alkali metal ion-substituted $\mathrm{LaMnO}_{3}$ were not studied till recently. We prepared $\mathrm{La}_{1-x} \mathrm{Na}_{x} \mathrm{MnO}_{3}, \mathrm{La}_{1-x} \mathrm{~K}_{x} \mathrm{MnO}_{3}$ oxides by $\mathrm{NaCl}$ or $\mathrm{KCl}$ flux method ${ }^{34}$. Epitaxial films of $\mathrm{Na}$ - and $\mathrm{K}$-doped $\mathrm{LaMnO}_{3}$ have been fabricated and their CMR properties have been studied. Na-substituted $\mathrm{LaMnO}_{3}$ shows $T_{c}$ of $310 \mathrm{~K}$ and at room temperature, over $75 \%$ CMR is observed at 6 tesla $^{35,36}$. A new phenomenon called charge ordering has been observed in the manganites at particular doping concentrations $(x=1 / 8,1 / 4,1 / 2)^{37}$. However, charge ordering is suppressed in the films due to substrate-lattice film strain effect which is discussed below.

\section{Modification of properties due to strained growth}

In some cases, thin film properties deviate from those of their bulk samples due to changes in the structure of the films. One of the important causes of such change originates from the lattice mismatch of the growing material with the substrate. When oxide films are deposited on a substrate with a lattice parameter smaller or larger than the film materials, they grow under strain. Though many researchers have speculated on reasons for such behaviour no clear understanding has as yet been arrived at. Rare earth nickelate polycrystalline samples, excluding $\mathrm{LaNiO}_{3}$ show metal-to-insulator transition ${ }^{38}$. Rare earth nickelate $\mathrm{RNiO}_{3}(\mathrm{R}=\mathrm{La}, \mathrm{Pr}, \mathrm{Nd}$ and $\mathrm{Sm})$ films grown on $\mathrm{LaAlO}_{3}(100), \mathrm{SrTiO}_{3}(100)$ and sapphire show variation in their transport properties. In these thin films the metal-to-insulator (MI) transition has been suppressed. Metallic behaviour for $\mathrm{PrNiO}_{3}$ film has been observed unlike in the bulk solid which shows metal-insulator transition. A detailed analysis of these films on different substrates (such as LAO, STO and sapphire) indicated the lattice mismatch as the main cause for the observed suppression of metal to insulator transition. A detailed investigation of $\mathrm{PrNiO}_{3}$ films by varying the thickness has confirmed the critical thickness that undergoes strain on the substrate ${ }^{22}$. This is analogous to the external pressure and increases the bond angle of $\mathrm{Ni}-\mathrm{O}-\mathrm{Ni}$ in turn increasing the bandwidth.

When $50 \%$ of $\mathrm{Nd}^{3+}$ ion is substituted for by $\mathrm{Sr}^{2+}$ ion in $\mathrm{NdMnO}_{3}$, equal amounts of $\mathrm{Mn}^{3+}$ and $\mathrm{Mn}^{4+}$ ions order in the lattice leading to charge-ordering phenomenon ${ }^{37} \cdot \mathrm{Ca}^{2+}$ ion-substituted $\mathrm{PrMnO}_{3}$ also showed charge-ordering behaviour. Epitaxial thin films of these charge-ordered materials did not show charge-ordering phenomenon. Due to 
lattice strain effect, the films crystallize in a more symmetric structure (pseudotetragonal instead of orthorhombic), and charge-ordering is suppressed. We have shown this by fabricating a series of Sr- and $\mathrm{Ca}$-doped $\mathrm{LnMnO}_{3}(\mathrm{Ln}=\mathrm{Pr}$ and $\mathrm{Nd})$ systems $^{39}$.

\section{Enhancement of magnetoresistance}

Manganites are considered potential candidates for magnetic read-head applications. The intrinsic magnetoresistance property of the manganites due to double exchange and other interactions cannot be exploited for the magnetic read-head technology, as this demands very high magnetic fields. Hence, the device structure should be engineered in such a way that low-field magnetoresistance can be achieved by extrinsic effects.

In order to increase the magnetoresistance of manganites, the multilayer technique has been employed. In most of these studies, large magnetoresistance was observed only at temperatures below $100 \mathrm{~K}$. Sandwiching the charge-ordered films between ferromagnetic metallic $\mathrm{La}_{0.7} \mathrm{Ca}_{0.3} \mathrm{MnO}_{3}$ has been proposed in order to enhance the magnetoresistance in manganites ${ }^{40,41}$. The internal field of the $\mathrm{La}_{0.7} \mathrm{Ca}_{0.3} \mathrm{MnO}_{3}$ and a small external magnetic field would be sufficient to obtain large magnetoresistance. A large magnetoresistance of $98 \%$ at $215 \mathrm{~K}$ has been obtained in these multilayers compared to $80 \% \mathrm{CMR}$ in $\mathrm{La}_{0.67} \mathrm{Ca}_{0.33} \mathrm{MnO}_{3}$ epitaxial film. This enhancement of $\mathrm{MR}$ effect has been verified by fabricating multilayers of $\mathrm{Pb}$ - and $\mathrm{Sr}$-substituted $\mathrm{LaMnO}_{3}$ with $\mathrm{Pr}_{0.7} \mathrm{Ca}_{0.3} \mathrm{MnO}_{3}$, and $\mathrm{Nd}_{0.5} \mathrm{Ca}_{0.5} \mathrm{MnO}_{3}$ as the charge-ordered spacer materials. Comparison has been made with $\mathrm{LaMnO}_{3}$ as the spacer layer where no double exchange can be induced since all the $\mathrm{Mn}$ ions are in the +3 state. In fact a decrease in magnetoresistance has been noticed in these multilayers as shown in figure 8 . From the

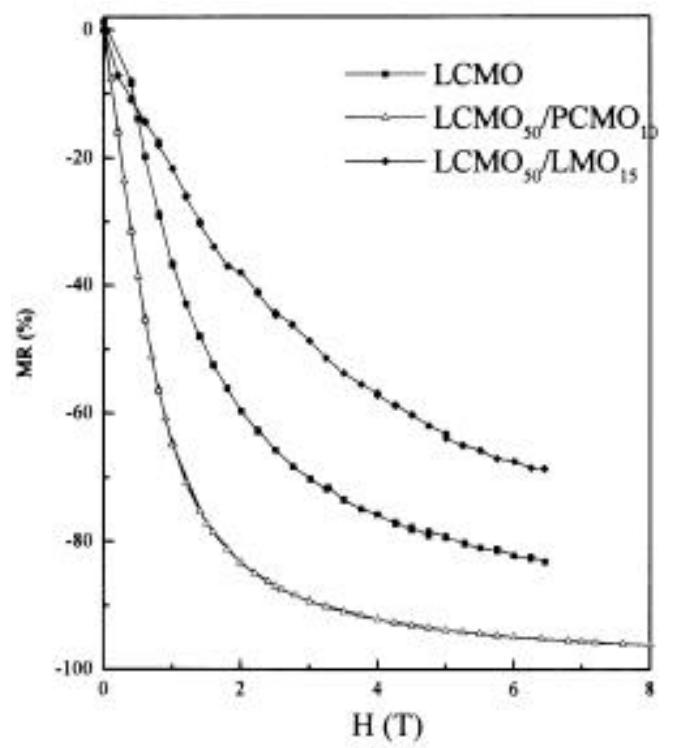

Figure 8. The plots of magnetoresistance as a function of applied magnetic field for the LCMO single layer film and LCMO/LNO, LCMO/PCMO multilayers. The curves represent the maximum observed magnetoresistance near their transition temperature. 
results it can be concluded that in order to increase the magnetoresistance we have to use a spacer layer that releases a greater number of conducting carriers.

In order to understand the magnetic and transport properties of the oxide multilayers of magnetic and metallic oxides, $\mathrm{La}_{0.6} \mathrm{~Pb}_{0.4} \mathrm{MnO}_{3} / \mathrm{La}_{4} \mathrm{BaCu}_{5} \mathrm{O}_{13}+\delta$ multilayers have also been fabricated. Here it is to be noted that both are metallic oxides. Interestingly no insulator-to-metal transition has been observed in these materials for thickness above 2 unit cells of $\mathrm{La}_{4} \mathrm{BaCu}_{5} \mathrm{O}_{13}+\delta$. Nevertheless they show ferromagnetic behaviour at low temperatures. The magnetic exchange coupling decreases monotonically in contrast to the metallic superlattices where an oscillatory exchange coupling is observed. The spins at the bilayer interface are disordered (canted) due to the copper and manganese superexchange interaction. The electrical transport has shown 2D nature in the absence of the magnetic field. A dimensionality crossover from 2D to 3D has been observed by the application of the magnetic field. It is not just the magnetic coupling between the ferromagnetic layers getting affected, the importance of the metallic coupling has been emphasized in the layered structure also ${ }^{42}$.

\section{Future prospects}

In recent times, superconductor $(\mathrm{S}) /$ ferromagnetic $(\mathrm{F})$ heterostructures have received much attention because of their rich physics and proposed applications. S/F multilayers show oscillatory dependence of $T_{c}$ with the thickness of the $\mathrm{S}$ layer or $\mathrm{F}$ layer. However, such experimental studies have not been available for high $T_{c}$ materials. Nevertheless, high $T_{c}$, CMR bilayers have been fabricated and it has been shown that they are best suited for microwave filters. Good quality high $T_{c}$ film can be used in biometric applications and microwave wave guides.

Although InSb films on CdTe and such semiconducting materials are grown by PLD, molecular beam epitaxy (MBE) is a superior technique for obtaining defect-free epilayers. Since PLD is a fast method, so far most studies were concerned with oxide films. However, vaporization of materials by pulsed laser is a general technique and in principle, almost all materials can be made into films by PLD.

PLD is thus a versatile technique to obtain epitaxial films of multicomponant oxide materials and, therefore, has become a standard experimental method to prepare films in general and high quality epitaxial films in particular for research purposes.

It is important to note that YBCO is grown in-situ under oxygen partial pressure. Reaction between oxygen and metal ions takes place in the plume. Similar reaction between molecules such as dinitrogen, hydrogen, ammonia, methane, acetylene, diborane can induce reactions leading to formation of compounds other than oxides. This aspect of PLD is open to research.

Metal films and their multilayers can be grown by the ablation of an oxide in hydrogen atmosphere. Tungsten metal film can be made by ablating $\mathrm{WO}_{3}$ in hydrogen.

Growth of binary and ternary nitride films is a distinct possibility. Besides making PLD films from stoichiometric nitrides, ablation of oxides in ammonia atmosphere can lead to metal nitride and metal oxynitride films. This follows from the ammonolysis of oxides into nitrides.

Thin films of transition metal sulphides and other chalcogenides are hardly studied. It is not perhaps difficult to make sulphide thin films by PLD. 
Growth of metal carbides can be attempted by the ablation of metal carbides or metal oxides in methane or ethane. Diborane is a gas that can be used to make metal boride films.

Besides making highly crystalline films on single crystal substrates, PLD has also been employed to make nano particles. $\mathrm{WO}_{3}$ particles of $200-300 \mathrm{~nm}$ grown by the PLD show good electrochromic effect. Similarly, heavily doped antimony tin oxide (ATO) by PLD can give both capacitive and electrochromic films ${ }^{43}$.

Therefore, PLD has good potential for use in making a variety of materials into films and nano particles.

\section{Acknowledgements}

Thanks are due to my co-workers, especially KM Satyalakshmi, Ms M Sahana and Mr A Venimadhav, who have contributed significantly to my efforts in the study of epitaxial oxide films by PLD. Continuous financial support from the Department of Science and Technology (DST), Government of India is gratefully acknowledged.

\section{References}

1. Hubler G K 1992 Mater. Res. Soc. Bull. 1725

2. Dijkkamp D, Venkatesan T, Wu X D, Shaheen S A, Jisrawi, N, Min-Lee, Y H, Mclean WL and Croft M 1987 Appl. Phys. Lett. 51619

3. Inam A, Hegde M S, Wu X D, Venkatesan T, England P, Miceli P F, Chase E W, Chang C C, Tarascon J-M and Wachtman J B 1988 Appl. Phys. Lett. 53908

4. Hegde M S, Satyalakshmi K M, Mallya R M, Rajeswari M and Zhang H 1994 J.Mater.Res. 9898

5. Triscone J M, Karkut M G, Antognazza L, Brunner O, and Fischer O 1989 Phys. Rev. Lett. 631016

6. Cuaudhari P, Koch R H, Laibowitz R B, McGuire T R and Gambino R J 1987 Phys. Rev. Lett. $\mathbf{5 8} 2684$

7. Rao C N R and Gopalakrishnan J 1997 New directions in solid state chemistry (Cambridge: University Press) p. 436

8. Easwar B K, Sarkar K, Kumar D, Hegde M S, Pinto R and Apte P R 1995 Electron. Lett. 31 374

9. Kumar D, Satyalakshmi K M, Manoharan S S and Hegde M S 1994 Bull. Mater. Sci. 17625

10. Pinto R, Apte P R, Hegde M S and Kumar D 1995 J. Appl. Phys. 774116

11. Pinto R, Apte P R, Adhi K P, Ogale S B, Kumar D and Hegde M S 1995 J. Appl. Phys. 78 5204

12. Hegde M S, Thomas B, Vasanthacharya N Y, Bhat S V, Srinivasu V V and Kumar N 1993 Phys. Rev. B48 6465

13. Rogers C T, Inam A, Hegde M S, Dutta B, Wu X D and Venkatesan T 1989 Appl. Phys. Lett. 552032

14. Satyalakshmi KM 1995 Studies on superconducting metallic and ferroelectric oxide thin films and their heterostructures grown by pulsed laser deposition, $\mathrm{Ph} \mathrm{D}$ thesis, Indian Institute of Science, Bangalore, p. 138

15. Wollman D A, Van Harlingen D J, Lee W C and Ginsberg D M 1993 Phys. Rev. Lett. 71 2134

16. Ioffe L B, Geshkenbeni V B, Feigel'man M V, Fauchere A L and Blatter G 1999 Nature (London) 398679

17. Ramesh R, Inam A, Chan W K, Wilkins B, Mayers K, Ramsching K, Hart D L and Tarascon J-M 1991 Science 252944

18. Satyalakshmi K M, Mallya R M, Ramanathan K V, Wu X D, Brainard B, Gautier D C, Vasanthacharya N Y and Hegde M S 1993 Appl. Phys. Lett. 621233 
19. Hegde M S, Satyalakshmi K M, Manoharan S S and Kumar D 1995 Mater. Sci. Eng. B32 239

20. Kumar D, Satyalakshmi K M, Hegde M S, Apte P R and Pinto R 1995 IEEE Trans. Appl. Superconductivity 53498

21. Venimadhav A, Herle P S, Vedvyas M, Sivakumara C and Hegde M S 1999 Appl. Phys. Lett. 751598

22. Venimadhav A, Chaitanyalekshmi I and Hegde M S 2002 Mater. Res. Bull. (Jan. issue) (in press)

23. Prasad K V R, Varma K B R, Raju A R, Satyalakshmi K M, Mallya R M and Hegde M S 1993 Appl. Phys. Lett. 631898

24. Satyalakshmi K M, Varma K B R and Hegde M S 1995 J. Appl. Phys. 78116

25. Jonker G H and van Santen J H 1950 Physica 16337

26. Von Humbolt R, Wecker B, Holzapfel B, Schulz L and Samwer K 1993 Phys. Rev. Lett. 71 1331

27. Jin H, Tiefel T H, McCormack M, Fastnacht R A and Ramesh R 1994 Science 264413

28. Ju L H, Kwon C, Li Q, Greene R L and Venkatesan T 1994 Appl. Phys. Lett. 652108

29. Manoharan S S, Vasanthacharya N Y, Hegde M S, Satyalakshmi K M, Prasad V and Subramanyam S V 1994 J. Appl. Phys. 763923

30. Satyalakshmi K M, Manoharan S S, Hegde M S, Prasad V and Subramanyam S V 1995 J. Appl. Phys. 786861

31. Sahana M, Satyalakshmi K M, Hegde M S, Prasad V and Subramanyam S V 1997 Mater. Res. Bull. 32831

32. Manoharan S S, Kumar D, Hegde M S, Satyalakshmi K M, Prasad V and Subramanyam S V 1995 J. Solid State Chem. 117420

33. Sahana M, Hegde M S, Vasanthacharya N Y, Prasad V and Subramanyam S V 1997 Appl. Phys. Lett. 712701

34. Singh R N, Shivakumara C, Vasanthacharya N Y, Subramanyam S, Hegde M S, Rajagopal H and Sequiera A 1998 J. Solid State Chem. 137191

35. Sahana M, Singh R N, Shivakumara C, Vasanthacharya N Y, Hegde M S, Prasad V and Subramanyam S V 1997 Appl. Phys. Lett. 702909

36. Sahana M, Hegde M S, Shivakumara C, Prasad V and Subramanyam S V 1999 J. Solid State Chem. 148342

37. Kuwahara H, Tomioka Y, Asamitsu A, Morimoto Y and Tokura Y 1995 Science 370961

38. Torrance J B, Lacorre P, Nazzal I, Ansaldo E J and Niedermayer C H 1992 Phys. Rev. B45 8209

39. Venimadhav A, Hegde M S, Rawat R and Das I 2001 J. Appl. Phys. 898057

40. Venimadhav A, Hegde M S, Prasad V and Subramanyam S V 2000 J. Phys. 332921

41. Venimadhav A, Hegde M S, Rawat R and Das I 2001 J. Alloys Compds. 326270

42. Venimadhav A, Hegde M S, Rawat R, Das, I, Paulose P L and Sampathkumaran E V 2001 Phys. Rev. B63 214404

43. Marcel C, Hegde M S, Rougier A, Maugy C, Guéry C and Tarascon J-M 2001 Electrochim. Acta 462097 